\title{
Kybernetika
}

\section{Sebastian Fuchs}

Multivariate copulas: Transformations, symmetry, order and measures of concordance

Kybernetika, Vol. 50 (2014), No. 5, 725-743

Persistent URL: http://dml.cz/dmlcz/144103

\section{Terms of use:}

(C) Institute of Information Theory and Automation AS CR, 2014

Institute of Mathematics of the Czech Academy of Sciences provides access to digitized documents strictly for personal use. Each copy of any part of this document must contain these Terms of use.

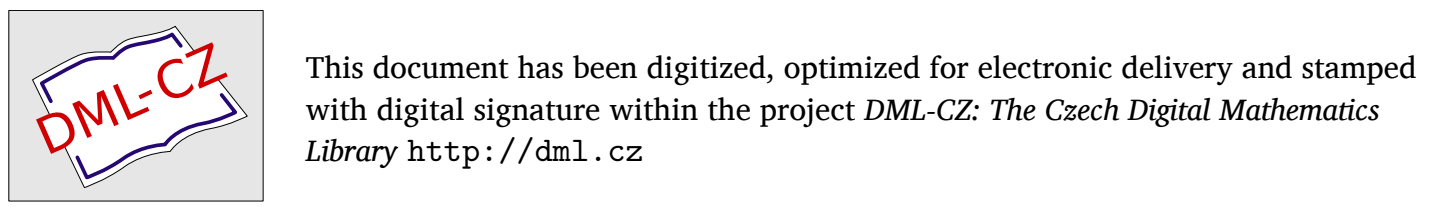




\title{
MULTIVARIATE COPULAS: TRANSFORMATIONS, SYMMETRY, ORDER AND MEASURES OF CONCORDANCE
}

\author{
Sebastian Fuchs
}

The present paper introduces a group of transformations on the collection of all multivariate copulas. The group contains a subgroup which is of particular interest since its elements preserve symmetry, the concordance order between two copulas and the value of every measure of concordance.

Keywords: multivariate copulas, transformations, symmetry, order, measures of concordance

Classification: $60 \mathrm{E} 05,62 \mathrm{E} 10,62 \mathrm{H} 20,20 \mathrm{C} 99$

\section{INTRODUCTION}

The present paper provides the construction of a group of transformations on the collection of all $d$-dimensional copulas. This group consists of $2^{d} d$ ! elements and it is of interest since many of its elements

- preserve symmetry of a copula, or

- preserve concordance order between two copulas, or

- preserve the value of every measure of concordance of a copula.

In fact, we show that a given transformation has one of these properties if and only if it has each of these properties, and that the collection of all transformations having these properties is a subgroup which consists of $2 d$ ! elements. We also show that the transformations preserving ordinary order between copulas form a subgroup as well, and that the group contains a transformation which turns every copula into its survival copula.

We thus extend parts of the results of Fuchs and Schmidt [3] who considered the case $d=2$ and used the group table and the lattice of subgroups. The case of general $d$ considered here requires a different reasoning and more abstract arguments.

The group of transformations of $d$-dimensional copulas considered here can be shown to be isomorphic to the group of symmetries on the $d$-dimensional hypercube considered by Taylor [5, 6, 7] with regard to measures of concordance. Using the group of transformations is perhaps more abstract than using the group of symmetries, but it avoids the use of results from measure and probability theory.

DOI: $10.14736 /$ kyb-2014-5-0725 
This paper is organized as follows: We first introduce some elementary transformations of copulas (Section 2), and then construct the smallest group containing these transformations (Section 3). We next show that the group contains a transformation which turns every copula into its survival copula (Section 4), and further identify the subgroups of transformations preserving symmetry and order properties of copulas (Section 5) and the value of every measure of concordance of a copula (Section 6). We finally sketch a probabilistic look at the results of this paper (Section 7).

Let $\mathrm{I}:=[0,1]$ and let $d$ be an integer $\geq 2$ which will be kept fixed throughout this paper.

\section{ELEMENTARY TRANSFORMATIONS ON COPULAS}

We are interested in the collection of all copulas and study transformations that map the set of copulas into itself. For the sake of a concise definition of a copula we consider, for $L \subseteq\{1, \ldots, d\}$, the map $\boldsymbol{\eta}_{L}: \mathrm{I}^{d} \times \mathrm{I}^{d} \rightarrow \mathrm{I}^{d}$ given by

$$
\left(\boldsymbol{\eta}_{L}(\mathbf{u}, \mathbf{v})\right)_{l}:= \begin{cases}u_{l} & l \in\{1, \ldots, d\} \backslash L \\ v_{l} & l \in L\end{cases}
$$

$l \in\{1, \ldots, d\}$, and put $\boldsymbol{\eta}_{i}:=\boldsymbol{\eta}_{\{i\}}$ for $i \in\{1, \ldots, d\}$.

A copula is a function $C: \mathrm{I}^{d} \rightarrow \mathrm{I}$ satisfying the following conditions:

(i) The inequality

$$
\sum_{L \subseteq\{1, \ldots, d\}}(-1)^{d-|L|} C\left(\boldsymbol{\eta}_{L}(\mathbf{u}, \mathbf{v})\right) \geq 0
$$

holds for all $\mathbf{u}, \mathbf{v} \in \mathrm{I}^{d}$ such that $\mathbf{u} \leq \mathbf{v}$.

(ii) The identity

$$
C\left(\boldsymbol{\eta}_{i}(\mathbf{u}, \mathbf{0})\right)=0
$$

holds for all $\mathbf{u} \in \mathrm{I}^{d}$ and all $i \in\{1, \ldots, d\}$.

(iii) The identity

$$
C\left(\boldsymbol{\eta}_{i}(\mathbf{1}, \mathbf{u})\right)=u_{i}
$$

holds for all $\mathbf{u} \in \mathrm{I}^{d}$ and all $i \in\{1, \ldots, d\}$.

We denote by $\mathcal{M}$ the collection of all functions $\mathrm{I}^{d} \rightarrow \mathbb{R}$ and by $\mathcal{C}$ the collection of all copulas. Then $\mathcal{M}$ is an ordered vector space under the coordinatewise defined linear operations and order relation, and $\mathcal{C}$ is a convex subset of $\mathcal{M}$. A map $\varphi: \mathcal{C} \rightarrow \mathcal{M}$ is said to be a transformation.

Let $\Phi$ denote the collection of all transformations $\mathcal{C} \rightarrow \mathcal{C}$ and define the composition $\circ: \Phi \times \Phi \rightarrow \Phi$ by letting $\left(\varphi_{1} \circ \varphi_{2}\right)(C):=\varphi_{1}\left(\varphi_{2}(C)\right)$. The composition is associative. The transformation $\iota \in \Phi$ given by $\iota(C):=C$ satisfies $\iota \circ \varphi=\varphi=\varphi \circ \iota$ for every $\varphi \in \Phi$ and is called the identity on $\mathcal{C}$. A transformation $\varphi \in \Phi$ is said to be an involution if $\varphi \circ \varphi=\iota$. Moreover, let $\Phi^{*} \subseteq \Phi$ be the set of all elements of $\Phi$ for which there exists an inverse element in $\Phi$. We thus obtain the following result: 
Lemma 2.1. $(\Phi, \circ)$ is a semigroup with neutral element $\iota$. Moreover, $\left(\Phi^{*}, \circ\right)$ is a subgroup of $\Phi$ that contains all involutions.

The term subgroup in Lemma 2.1 means that $\left(\Phi^{*}, \circ\right)$ is a subsemigroup of $(\Phi, \circ)$ and a group.

We now introduce two elementary transformations, transpositions and partial reflections, on which we will focus our attention in the subsequent sections.

For $i, j \in\{1, \ldots, d\}$ with $i \neq j$ we define the map $\pi_{i, j}: \mathcal{C} \rightarrow \mathcal{M}$ by letting

$$
\left(\pi_{i, j}(C)\right)(\mathbf{u}):=C\left(\boldsymbol{\eta}_{\{i, j\}}\left(\mathbf{u}, u_{j} \mathbf{e}_{i}+u_{i} \mathbf{e}_{j}\right)\right)
$$

and, for $k \in\{1, \ldots, d\}$, the map $\nu_{k}: \mathcal{C} \rightarrow \mathcal{M}$ by letting

$$
\left(\nu_{k}(C)\right)(\mathbf{u}):=C\left(\boldsymbol{\eta}_{k}(\mathbf{u}, \mathbf{1})\right)-C\left(\boldsymbol{\eta}_{k}(\mathbf{u}, \mathbf{1}-\mathbf{u})\right)
$$

$\pi_{i, j}$ is called a transposition, and $\nu_{k}$ is called a partial reflection.

It turns out that transpositions and partial reflections map the collection of all copulas into itself.

Lemma 2.2. Every transposition and every partial reflection is in $\Phi$. Moreover, transpositions and partial reflections are involutions and hence in $\Phi^{*}$.

Pr o of . Consider $C \in \mathcal{C}$. It is evident that $\pi_{i, j}(C)$ is a copula for every $i, j \in\{1, \ldots, d\}$ with $i \neq j$. Now, let $k \in\{1, \ldots, d\}$. We prove that $\nu_{k}(C)$ is a copula as well. To this end, consider first $\mathbf{u}, \mathbf{v} \in \mathrm{I}^{d}$ such that $\mathbf{u} \leq \mathbf{v}$. We then obtain

$$
\boldsymbol{\eta}_{k}\left(\mathbf{u},\left(1-v_{k}\right) \mathbf{e}_{k}\right) \leq \boldsymbol{\eta}_{k}\left(\mathbf{v},\left(1-u_{k}\right) \mathbf{e}_{k}\right)
$$

and hence

$$
\begin{aligned}
& \sum_{L \subseteq\{1, \ldots, d\}}(-1)^{d-|L|}\left(\nu_{k}(C)\right)\left(\boldsymbol{\eta}_{L}(\mathbf{u}, \mathbf{v})\right) \\
= & \sum_{L \subseteq\{1, \ldots, d\}}(-1)^{d-|L|}\left[C\left(\boldsymbol{\eta}_{k}\left(\boldsymbol{\eta}_{L}(\mathbf{u}, \mathbf{v}), \mathbf{1}\right)\right)-C\left(\boldsymbol{\eta}_{k}\left(\boldsymbol{\eta}_{L}(\mathbf{u}, \mathbf{v}), \mathbf{1}-\boldsymbol{\eta}_{L}(\mathbf{u}, \mathbf{v})\right)\right)\right] \\
= & -\sum_{L \subseteq\{1, \ldots, d\}}(-1)^{d-|L|} C\left(\boldsymbol{\eta}_{k}\left(\boldsymbol{\eta}_{L}(\mathbf{u}, \mathbf{v}), \mathbf{1}-\boldsymbol{\eta}_{L}(\mathbf{u}, \mathbf{v})\right)\right) \\
= & -\sum_{L \subseteq\{1, \ldots, d\} \backslash\{k\}}(-1)^{d-|L|} C\left(\boldsymbol{\eta}_{L \cup\{k\}}\left(\mathbf{u}, \sum_{l \in L} v_{l} \mathbf{e}_{l}+\left(1-u_{k}\right) \mathbf{e}_{k}\right)\right) \\
& -\sum_{L \subseteq\{1, \ldots, d\} \backslash\{k\}}(-1)^{d-|L \cup\{k\}|} C\left(\boldsymbol{\eta}_{L \cup\{k\}}\left(\mathbf{u}, \sum_{l \in L} v_{l} \mathbf{e}_{l}+\left(1-v_{k}\right) \mathbf{e}_{k}\right)\right) \\
= & \sum_{L \subseteq\{1, \ldots, d\} \backslash\{k\}}(-1)^{d-|L \cup\{k\}|} C\left(\boldsymbol{\eta}_{L \cup\{k\}}\left(\mathbf{u}, \boldsymbol{\eta}_{k}\left(\mathbf{v},\left(1-u_{k}\right) \mathbf{e}_{k}\right)\right)\right)
\end{aligned}
$$




$$
\begin{aligned}
& +\sum_{L \subseteq\{1, \ldots, d\} \backslash\{k\}}(-1)^{d-|L|} C\left(\boldsymbol{\eta}_{L}\left(\boldsymbol{\eta}_{k}\left(\mathbf{u},\left(1-v_{k}\right) \mathbf{e}_{k}\right), \mathbf{v}\right)\right) \\
= & \sum_{L \subseteq\{1, \ldots, d\} \backslash\{k\}}(-1)^{d-|L \cup\{k\}|} C\left(\boldsymbol{\eta}_{L \cup\{k\}}\left(\boldsymbol{\eta}_{k}\left(\mathbf{u},\left(1-v_{k}\right) \mathbf{e}_{k}\right), \boldsymbol{\eta}_{k}\left(\mathbf{v},\left(1-u_{k}\right) \mathbf{e}_{k}\right)\right)\right) \\
& +\sum_{L \subseteq\{1, \ldots, d\} \backslash\{k\}}(-1)^{d-|L|} C\left(\boldsymbol{\eta}_{L}\left(\boldsymbol{\eta}_{k}\left(\mathbf{u},\left(1-v_{k}\right) \mathbf{e}_{k}\right), \boldsymbol{\eta}_{k}\left(\mathbf{v},\left(1-u_{k}\right) \mathbf{e}_{k}\right)\right)\right) \\
= & \sum_{L \subseteq\{1, \ldots, d\}}(-1)^{d-|L|} C\left(\boldsymbol{\eta}_{L}\left(\boldsymbol{\eta}_{k}\left(\mathbf{u},\left(1-v_{k}\right) \mathbf{e}_{k}\right), \boldsymbol{\eta}_{k}\left(\mathbf{v},\left(1-u_{k}\right) \mathbf{e}_{k}\right)\right)\right) \\
\geq & 0
\end{aligned}
$$

which proves (i). The proofs of (ii) and (iii) are straightforward.

Furthermore, it is evident that every transposition is an involution. Consider again $k \in\{1, \ldots, d\}$ and $C \in \mathcal{C}$. Since $C$ is a copula, we obtain

$$
\begin{aligned}
&\left(\nu_{k}\left(\nu_{k}(C)\right)\right)(\mathbf{u}) \\
&=\left(\nu_{k}(C)\right)\left(\boldsymbol{\eta}_{k}(\mathbf{u}, \mathbf{1})\right)-\left(\nu_{k}(C)\right)\left(\boldsymbol{\eta}_{k}(\mathbf{u}, \mathbf{1}-\mathbf{u})\right) \\
&= C\left(\boldsymbol{\eta}_{k}\left(\boldsymbol{\eta}_{k}(\mathbf{u}, \mathbf{1}), \mathbf{1}\right)\right)-C\left(\boldsymbol{\eta}_{k}\left(\boldsymbol{\eta}_{k}(\mathbf{u}, \mathbf{1}), \mathbf{1}-\boldsymbol{\eta}_{k}(\mathbf{u}, \mathbf{1})\right)\right) \\
&-C\left(\boldsymbol{\eta}_{k}\left(\boldsymbol{\eta}_{k}(\mathbf{u}, \mathbf{1}-\mathbf{u}), \mathbf{1}\right)\right)+C\left(\boldsymbol{\eta}_{k}\left(\boldsymbol{\eta}_{k}(\mathbf{u}, \mathbf{1}-\mathbf{u}), \mathbf{1}-\boldsymbol{\eta}_{k}(\mathbf{u}, \mathbf{1}-\mathbf{u})\right)\right) \\
&= C\left(\boldsymbol{\eta}_{k}(\mathbf{u}, \mathbf{1})\right)-C\left(\boldsymbol{\eta}_{k}(\mathbf{u}, \mathbf{0})\right)-C\left(\boldsymbol{\eta}_{k}(\mathbf{u}, \mathbf{1})\right)+C(\mathbf{u}) \\
&= C(\mathbf{u}) .
\end{aligned}
$$

Therefore, every partial reflection is an involution.

Lemma 2.2 states that every transposition and every partial reflection is in $\Phi^{*}$. Thus, there exists a smallest subgroup of $\Phi$ containing all transpositions, a smallest subgroup of $\Phi$ containing all partial reflections, and a smallest subgroup of $\Phi$ containing all transpositions and all partial reflections.

\section{THE GROUP $\Gamma$}

In this section we construct the smallest subgroup of $\Phi$ that contains all transpositions, the smallest subgroup of $\Phi$ that contains all partial reflections and the smallest subgroup of $\Phi$ that contains all transpositions and all partial reflections.

For the composition of $m \in \mathbb{N}$ transformations $\varphi_{l} \in \Phi, l \in\{1, \ldots, m\}$, we write

$$
\bigcirc_{l=1}^{m} \varphi_{l}:= \begin{cases}\iota & m=0 \\ \varphi_{m} \circ \bigcirc_{l=1}^{m-1} \varphi_{l} & \text { otherwise }\end{cases}
$$

and, for $L=\{1, \ldots, m\}$ and a set of pairwise commuting $\varphi_{l} \in \Phi, l \in\{1, \ldots, m\}$, we put

$$
\bigcirc_{l \in L} \varphi_{l}:=\bigcirc_{l=1}^{m} \varphi_{l}
$$


Recall that the center of a group is the set of elements that commute with every element of the group, and the center is a subgroup.

Let us first consider the smallest subgroup of $\Phi$ that contains all transpositions. A transformation is called a permutation if it can be expressed as a finite composition of transpositions. Let $\Gamma^{\pi}$ be the set of all permutations.

Theorem 3.1. $\Gamma^{\pi}$ is the smallest subgroup of $\Phi$ containing all transpositions. Moreover, $\left|\Gamma^{\pi}\right|=d$ !. If $d=2$, then this subgroup is commutative; if $d \geq 3$, then its center is trivial.

Proof. First of all, since every composition of two permutations is a permutation, Lemma 2.1 implies that $\Gamma^{\pi}$ is a subsemigroup of $\Phi$ with neutral element $\iota$. Now, let $\pi \in \Gamma^{\pi}$. Then $\pi$ can be expressed as a finite composition of transpositions. Let $\pi^{*}$ be the composition of the same transpositions arranged in reverse order, then $\pi^{*} \in \Gamma^{\pi}$ and Lemma 2.2 yields

$$
\pi \circ \pi^{*}=\iota=\pi^{*} \circ \pi .
$$

Thus, $\Gamma^{\pi}$ is a subgroup of $\Phi$. A permutation is a transformation that rearranges the arguments of a copula. Since there exist $d$ ! possibilities to rearrange $d$ arguments, we hence obtain the cardinality of $\Gamma^{\pi}$.

For $d=2$, we have $\Gamma^{\pi}=\left\{\iota, \pi_{1,2}\right\}$ which shows that the elements of $\Gamma^{\pi}$ commute. Assume now that $d \geq 3$. To prove that the center of $\Gamma^{\pi}$ is trivial, consider $\pi \in \Gamma^{\pi} \backslash\{\iota\}$ and a copula $C \in \mathcal{C}$. Since $\pi \neq \iota$, there exist $k, l \in\{1, \ldots, d\}$ with $k \neq l$ such that the $k$ th coordinate of $C$ is the $l$ th coordinate of $\pi(C)$. Moreover, for $j \in\{1, \ldots, d\}$, consider the function $D_{j}: \mathrm{I}^{d} \rightarrow \mathbb{R}$ given by

$$
D_{j}(\mathbf{u}):=\prod_{i=1}^{d} u_{i}+\prod_{i=1, i \neq j}^{d}\left(1-u_{i}\right) \prod_{i=1}^{d} u_{i} .
$$

For every $j \in\{1, \ldots, d\} D_{j}$ is a copula; indeed for every $\mathbf{u}, \mathbf{v} \in \mathrm{I}^{d}$ such that $\mathbf{u} \leq \mathbf{v}$ we obtain

$$
\sum_{L \subseteq\{1, \ldots, d\}}(-1)^{d-|L|} D_{j}\left(\boldsymbol{\eta}_{L}(\mathbf{u}, \mathbf{v})\right)=\left[1+\prod_{i=1, i \neq j}^{d}\left(1-v_{i}-u_{i}\right)\right] \prod_{i=1}^{d}\left(v_{i}-u_{i}\right) \geq 0
$$

which proves (i); condition (ii) is obvious and (iii) follows from $d \geq 3$. Then we obtain

$$
\pi\left(D_{k}\right)=D_{l}
$$

and for some $m \in\{1, \ldots, d\} \backslash\{k, l\}$ (which exists since $d \geq 3$ ) we further obtain

$$
\left(\pi_{l m} \circ \pi \circ \pi_{l m}\right)\left(D_{k}\right)=\left(\pi_{l m} \circ \pi\right)\left(D_{k}\right)=\pi_{l m}\left(D_{l}\right)=D_{m} \neq D_{l}=\pi\left(D_{k}\right) .
$$

This yields $\pi \circ \pi_{l m} \neq \pi_{l m} \circ \pi$. Thus, $\{\iota\}$ is the center of $\Gamma^{\pi}$.

Now, let us consider the smallest subgroup of $\Phi$ that contains all partial reflections. A transformation is called a reflection if it can be expressed as a finite composition of partial reflections. Let $\Gamma^{\nu}$ be the set of all reflections. 
Lemma 3.2. The identity $\nu_{k} \circ \nu_{l}=\nu_{l} \circ \nu_{k}$ holds for all $k, l \in\{1, \ldots, d\}$.

Proof. Let $k, l \in\{1, \ldots, d\}, k \neq l$, and $C \in \mathcal{C}$. Then

$$
\begin{aligned}
\left(\nu_{k}\left(\nu_{l}(C)\right)\right)(\mathbf{u}) & \left(\nu_{l}(C)\right)\left(\boldsymbol{\eta}_{k}(\mathbf{u}, \mathbf{1})\right)-\left(\nu_{l}(C)\right)\left(\boldsymbol{\eta}_{k}(\mathbf{u}, \mathbf{1}-\mathbf{u})\right) \\
= & C\left(\boldsymbol{\eta}_{l}\left(\boldsymbol{\eta}_{k}(\mathbf{u}, \mathbf{1}), \mathbf{1}\right)\right)-C\left(\boldsymbol{\eta}_{l}\left(\boldsymbol{\eta}_{k}(\mathbf{u}, \mathbf{1}), \mathbf{1}-\boldsymbol{\eta}_{k}(\mathbf{u}, \mathbf{1})\right)\right) \\
& -C\left(\boldsymbol{\eta}_{l}\left(\boldsymbol{\eta}_{k}(\mathbf{u}, \mathbf{1}-\mathbf{u}), \mathbf{1}\right)\right)+C\left(\boldsymbol{\eta}_{l}\left(\boldsymbol{\eta}_{k}(\mathbf{u}, \mathbf{1}-\mathbf{u}), \mathbf{1}-\boldsymbol{\eta}_{k}(\mathbf{u}, \mathbf{1}-\mathbf{u})\right)\right) \\
= & C\left(\boldsymbol{\eta}_{\{k, l\}}(\mathbf{u}, \mathbf{1})\right)-C\left(\boldsymbol{\eta}_{\{k, l\}}\left(\mathbf{u}, 1 \mathbf{e}_{k}+\left(1-u_{l}\right) \mathbf{e}_{l}\right)\right)-C\left(\boldsymbol{\eta}_{\{k, l\}}\left(\mathbf{u},\left(1-u_{k}\right) \mathbf{e}_{k}+1 \mathbf{e}_{l}\right)\right) \\
& +C\left(\boldsymbol{\eta}_{\{k, l\}}(\mathbf{u}, \mathbf{1}-\mathbf{u})\right)
\end{aligned}
$$

and hence $\nu_{k} \circ \nu_{l}=\nu_{l} \circ \nu_{k}$. Since every partial reflection is an involution (Lemma 2.2 this proves the assertion.

Thus, a reflection can be expressed as a finite composition of partial reflections that includes every partial reflection at most once.

Example 3.3. The function $D: \mathrm{I}^{d} \rightarrow \mathbb{R}$ given by

$$
D(\mathbf{u}):=\prod_{i=1}^{d} u_{i}+\prod_{i=1}^{d} u_{i}^{2}\left(1-u_{i}\right)
$$

is a copula (see Dolati \& Úbeda-Flores [2, Example 4.2]) which satisfies $\pi(D)=D$ for all $\pi \in \Gamma^{\pi}$ and $\tilde{\nu}(D) \neq \bar{\nu}(D)$ for all $\tilde{\nu}, \bar{\nu} \in \Gamma^{\nu}$ such that $\tilde{\nu} \neq \bar{\nu}$.

Theorem 3.4. $\Gamma^{\nu}$ is the smallest subgroup of $\Phi$ containing all partial reflections. Moreover, $\left|\Gamma^{\nu}\right|=2^{d}$. This subgroup is commutative and each of its elements is an involution.

Pr o of. Since every composition of two reflections is a reflection, Lemma 2.1 implies that $\Gamma^{\nu}$ is a subsemigroup of $\Phi$ with neutral element $\iota$. Moreover, since partial reflections commute and are involutions, it follows that in fact all reflections commute and are involutions. In particular, $\Gamma^{\nu}$ is a subgroup of $\Phi$.

Since every reflection can be expressed as a composition of partial reflections that includes every partial reflection at most once, the cardinality of $\Gamma^{\nu}$ is at most $2^{d}$. That $\left|\Gamma^{\nu}\right|=2^{d}$ then follows from Example 3.3 since the application of the reflections in $\Gamma^{\nu}$ to $D$ produces $2^{d}$ distinct copulas.

Finally, let us consider the smallest subgroup of $\Phi$ that contains all transpositions and all partial reflections. A transformation is called a symmetry if it can be expressed as a composition of a permutation and a reflection. Let $\Gamma$ be the set of all symmetries.

We need the following lemma which includes results for the composition of a transposition and a partial reflection which is again a transformation in $\Phi$. 
Lemma 3.5. Let $i, j \in\{1, \ldots, d\}$ with $i \neq j$ and $k \in\{1, \ldots, d\} \backslash\{i, j\}$. Then

(1) $\pi_{i, j} \circ \nu_{j}=\nu_{i} \circ \pi_{i, j}$

(2) $\pi_{i, j} \circ \nu_{i}=\nu_{j} \circ \pi_{i, j}$

(3) $\pi_{i, j} \circ \nu_{k}=\nu_{k} \circ \pi_{i, j}$

In particular, $\pi_{i, j}$ commutes neither with $\nu_{i}$ nor with $\nu_{j}$.

Proof. Let $C \in \mathcal{C}$. Then, we obtain

$$
\begin{aligned}
\left(\pi_{i, j}\left(\nu_{j}(C)\right)\right)(\mathbf{u})= & \left(\nu_{j}(C)\right)\left(\boldsymbol{\eta}_{\{i, j\}}\left(\mathbf{u}, u_{j} \mathbf{e}_{i}+u_{i} \mathbf{e}_{j}\right)\right) \\
= & C\left(\boldsymbol{\eta}_{j}\left(\boldsymbol{\eta}_{\{i, j\}}\left(\mathbf{u}, u_{j} \mathbf{e}_{i}+u_{i} \mathbf{e}_{j}\right), \mathbf{1}\right)\right) \\
& -C\left(\boldsymbol{\eta}_{j}\left(\boldsymbol{\eta}_{\{i, j\}}\left(\mathbf{u}, u_{j} \mathbf{e}_{i}+u_{i} \mathbf{e}_{j}\right), \mathbf{1}-\boldsymbol{\eta}_{\{i, j\}}\left(\mathbf{u}, u_{j} \mathbf{e}_{i}+u_{i} \mathbf{e}_{j}\right)\right)\right) \\
= & C\left(\boldsymbol{\eta}_{\{i, j\}}\left(\mathbf{u}, u_{j} \mathbf{e}_{i}+1 \mathbf{e}_{j}\right)\right)-C\left(\boldsymbol{\eta}_{\{i, j\}}\left(\mathbf{u}, u_{j} \mathbf{e}_{i}+\left(1-u_{i}\right) \mathbf{e}_{j}\right)\right) \\
= & \left(\pi_{i, j}(C)\right)\left(\boldsymbol{\eta}_{i}(\mathbf{u}, \mathbf{1})\right)-\left(\pi_{i, j}(C)\right)\left(\boldsymbol{\eta}_{i}(\mathbf{u}, \mathbf{1}-\mathbf{u})\right) \\
= & \left(\nu_{i}\left(\pi_{i, j}(C)\right)\right)(\mathbf{u}) .
\end{aligned}
$$

This proves (1). Analogously, we obtain (2). Moreover

$$
\begin{aligned}
&\left(\pi_{i, j}\left(\nu_{k}(C)\right)\right)(\mathbf{u}) \\
&=\left(\nu_{k}(C)\right)\left(\boldsymbol{\eta}_{\{i, j\}}\left(\mathbf{u}, u_{j} \mathbf{e}_{i}+u_{i} \mathbf{e}_{j}\right)\right) \\
&=C\left(\boldsymbol{\eta}_{k}\left(\boldsymbol{\eta}_{\{i, j\}}\left(\mathbf{u}, u_{j} \mathbf{e}_{i}+u_{i} \mathbf{e}_{j}\right), \mathbf{1}\right)\right) \\
& \quad-C\left(\boldsymbol{\eta}_{k}\left(\boldsymbol{\eta}_{\{i, j\}}\left(\mathbf{u}, u_{j} \mathbf{e}_{i}+u_{i} \mathbf{e}_{j}\right), \mathbf{1}-\boldsymbol{\eta}_{\{i, j\}}\left(\mathbf{u}, u_{j} \mathbf{e}_{i}+u_{i} \mathbf{e}_{j}\right)\right)\right) \\
&=C\left(\boldsymbol{\eta}_{\{i, j, k\}}\left(\mathbf{u}, u_{j} \mathbf{e}_{i}+u_{i} \mathbf{e}_{j}+1 \mathbf{e}_{k}\right)\right)-C\left(\boldsymbol{\eta}_{\{i, j, k\}}\left(\mathbf{u}, u_{j} \mathbf{e}_{i}+u_{i} \mathbf{e}_{j}+\left(1-u_{k}\right) \mathbf{e}_{k}\right)\right) \\
&=\left(\pi_{i, j}(C)\right)\left(\boldsymbol{\eta}_{k}(\mathbf{u}, \mathbf{1})\right)-\left(\pi_{i, j}(C)\right)\left(\boldsymbol{\eta}_{k}(\mathbf{u}, \mathbf{1}-\mathbf{u})\right) \\
&=\left(\nu_{k}\left(\pi_{i, j}(C)\right)\right)(\mathbf{u}) .
\end{aligned}
$$

This proves $(3)$.

The following lemma comprises results for the composition of a permutation and a reflection, and it also shows, that the intersection of the subgroups $\Gamma^{\pi}$ and $\Gamma^{\nu}$ is trivial.

Lemma 3.6. (1) For every $\pi \in \Gamma^{\pi}$ and every $\nu \in \Gamma^{\nu}$ there exists some $\widehat{\pi} \in \Gamma^{\pi}$ and some $\widehat{\nu} \in \Gamma^{\nu}$ such that

$$
\pi \circ \nu=\widehat{\nu} \circ \widehat{\pi} .
$$

(2) For every $\pi \in \Gamma^{\pi}$ and every $\nu \in \Gamma^{\nu}$ there exists some $\widehat{\pi} \in \Gamma^{\pi}$ and some $\widehat{\nu} \in \Gamma^{\nu}$ such that

$$
\nu \circ \pi=\widehat{\pi} \circ \widehat{\nu}
$$

(3) $\Gamma^{\pi} \cap \Gamma^{\nu}=\{\iota\}$. 
Proof. Assertions (1) and (2) immediately follow from Lemma 3.5. We now prove (3). It is obvious that $\iota \in \Gamma^{\pi} \cap \Gamma^{\nu}$. Assume that there exists some $\gamma \in \Gamma^{\pi} \cap \Gamma^{\nu}$ such that $\gamma \neq \iota$ and consider the copula $D$ discussed in Example 3.3 . Since $\gamma \in \Gamma^{\pi}$ we have $\gamma(D)=D$, and since $\gamma \in \Gamma^{\nu}$ and $\gamma \neq \iota$ we have $\gamma(D) \neq \iota(D)=D$. Thus, $\Gamma^{\pi} \cap \Gamma^{\nu}=\{\iota\}$.

Lemma 3.6 yields

$$
\begin{aligned}
\Gamma & =\left\{\varphi \in \Phi \mid \varphi=\pi \circ \nu \text { for some } \pi \in \Gamma^{\pi} \text { and some } \nu \in \Gamma^{\nu}\right\} \\
& =\left\{\varphi \in \Phi \mid \varphi=\nu \circ \pi \text { for some } \pi \in \Gamma^{\pi} \text { and some } \nu \in \Gamma^{\nu}\right\}
\end{aligned}
$$

and the representation of a symmetry in terms of a permutation and a reflection is unique which is again a consequence of Lemma 3.6 .

Lemma 3.7. (1) For every $\gamma \in \Gamma$ there exist unique $\pi \in \Gamma^{\pi}$ and $\nu \in \Gamma^{\nu}$ such that $\gamma=\pi \circ \nu$.

(2) For every $\gamma \in \Gamma$ there exist unique $\pi \in \Gamma^{\pi}$ and $\nu \in \Gamma^{\nu}$ such that $\gamma=\nu \circ \pi$.

The reflection which can be expressed as a composition of partial reflections that includes every partial reflection exactly once is called total reflection. We denote the total reflection by $\tau$ which differs from the notation used in Fuchs \& Schmidt [3]. The total reflection is an involution and we have

$$
\tau=\bigcirc_{l=1}^{d} \nu_{l}
$$

Moreover, set

$$
\Gamma^{\tau}:=\{\iota, \tau\}
$$

Theorem 3.8. $\Gamma$ is the smallest subgroup of $\Phi$ containing all transpositions and all partial reflections. Moreover, $|\Gamma|=d ! 2^{d}$ and the center of $\Gamma$ is $\Gamma^{\tau}$.

Proof. First of all, consider $\widehat{\gamma}, \bar{\gamma} \in \Gamma$. Then Lemma 3.7 provides the existence of unique $\widehat{\pi}, \bar{\pi} \in \Gamma^{\pi}$ and $\widehat{\nu}, \bar{\nu} \in \Gamma^{\nu}$ such that $\widehat{\gamma}=\widehat{\pi} \circ \widehat{\nu}$ and $\bar{\gamma}=\bar{\pi} \circ \bar{\nu}$. With regard to Lemma 3.6 there exists some $\pi \in \Gamma^{\pi}$ and some $\nu \in \Gamma^{\nu}$ such that $\widehat{\nu} \circ \bar{\pi}=\pi \circ \nu$. This yields

$$
\widehat{\gamma} \circ \bar{\gamma}=\widehat{\pi} \circ \widehat{\nu} \circ \bar{\pi} \circ \bar{\nu}=\widehat{\pi} \circ \pi \circ \nu \circ \bar{\nu}
$$

Since $\widehat{\pi} \circ \pi \in \Gamma^{\pi}$ and $\nu \circ \bar{\nu} \in \Gamma^{\nu}$ we obtain $\widehat{\gamma} \circ \bar{\gamma} \in \Gamma$, and thus, Lemma 2.1 implies that $\Gamma$ is a subsemigroup of $\Phi$ with neutral element $\iota$. Furthermore, let $\widehat{\pi}^{*} \in \Gamma^{\pi}$ be the inverse element of $\widehat{\pi}$ and let $\widehat{\nu}^{*} \in \Gamma^{\nu}$ be the inverse element of $\widehat{\nu}$. Set $\widehat{\gamma}^{*}:=\widehat{\nu}^{*} \circ \widehat{\pi}^{*}$. Then $\widehat{\gamma}^{*} \in \Gamma$ and we obtain

$$
\widehat{\gamma} \circ \widehat{\gamma}^{*}=\widehat{\pi} \circ \widehat{\nu} \circ \widehat{\nu}^{*} \circ \widehat{\pi}^{*}=\iota=\widehat{\nu}^{*} \circ \widehat{\pi}^{*} \circ \widehat{\pi} \circ \widehat{\nu}=\widehat{\gamma}^{*} \circ \widehat{\gamma}
$$

Thus, $\Gamma$ is a subgroup of $\Phi$ and since $\Gamma^{\pi} \cap \Gamma^{\nu}=\{\iota\}$ we obtain $|\Gamma|=\left|\Gamma^{\pi}\right| \cdot\left|\Gamma^{\nu}\right|=d ! 2^{d}$. 
We now prove that the center $Z(\Gamma)$ of $\Gamma$ is $\Gamma^{\tau}$. For the case $d=2$ the proof is given in Fuchs \& Schmidt [3. Further, let $d \geq 3$. First of all, Lemma 3.5 implies

$\pi_{i, j} \circ \tau=\pi_{i, j} \circ \bigcirc_{l=1}^{d} \nu_{l}=\bigcirc_{l \in\{1, \ldots, d\} \backslash\{i, j\}} \nu_{l} \circ \pi_{i, j} \circ \nu_{i} \circ \nu_{j}=\bigcirc_{l \in\{1, \ldots, d\} \backslash\{i, j\}} \nu_{l} \circ \nu_{j} \circ \nu_{i} \circ \pi_{i, j}=\tau \circ \pi_{i, j}$

for all $i, j \in\{1, \ldots, d\}$ with $i \neq j$, and hence $\pi \circ \tau=\tau \circ \pi$ for every $\pi \in \Gamma^{\pi}$. Since $\tau$ commutes with every reflection we obtain $\Gamma^{\tau} \subseteq Z(\Gamma)$. Moreover, since the center of $\Gamma^{\pi}$ is trivial, we have $Z(\Gamma) \cap \Gamma^{\pi}=\{\iota\}$, and thus, the subgroup $Z(\Gamma)$ lies in the largest subgroup $\Lambda \subseteq \Gamma$ of $\Gamma$ which satisfies $\Lambda \cap \Gamma^{\pi}=\{\iota\}$. We now prove that $\Gamma^{\nu}$ is the largest subgroup satisfying this property. Note that $\Gamma^{\nu} \cap \Gamma^{\pi}=\{\iota\}$ and assume that there exists a subgroup $\Lambda \subseteq \Gamma$ which satisfies $\Gamma^{\nu} \subset \Lambda$ and $\Lambda \cap \Gamma^{\pi}=\{\iota\}$. Then, there exists a $\gamma \in \Lambda$ such that $\gamma=\pi \circ \nu$ with unique $\pi \in \Gamma^{\pi} \backslash\{\iota\}$ and $\nu \in \Gamma^{\nu}$. Let $\nu^{*} \in \Lambda$ be the inverse element of $\nu$. Since $\Lambda$ is a subgroup we obtain

$$
\pi=\pi \circ \nu \circ \nu^{*}=\gamma \circ \nu^{*} \in \Lambda
$$

which contradicts to $\Lambda \cap \Gamma^{\pi}=\{\iota\}$. Thus, $Z(\Gamma) \subseteq \Gamma^{\nu}$. Now, let $\nu \in \Gamma^{\nu} \backslash \Gamma^{\tau}$. Then there exists some $K \subseteq\{1, \ldots, d\}$ with $1 \leq|K| \leq d-1$ such that $\nu=\bigcirc_{l \in K} \nu_{l}$. Moreover, choose $p \in K$ and $q \in\{1, \ldots, d\} \backslash K$. Lemma 3.5 then yields

$$
\begin{aligned}
\pi_{p, q} \circ \nu & =\pi_{p, q} \circ \bigcirc_{l \in K} \nu_{l} \\
& =\pi_{p, q} \circ \bigcirc_{l \in K \backslash\{p\}} \nu_{l} \circ \nu_{p} \\
& =\bigcirc_{l \in K \backslash\{p\}} \nu_{l} \circ \pi_{p, q} \circ \nu_{p} \\
& =\bigcirc_{l \in K \backslash\{p\}} \nu_{l} \circ \nu_{q} \circ \pi_{p, q} .
\end{aligned}
$$

Further, consider the copula $D$ discussed in Example 3.3. We then obtain

$$
\begin{aligned}
\left(\pi_{p, q} \circ \nu\right)(D) & =\left(\bigcirc_{l \in K \backslash\{p\}} \nu_{l} \circ \nu_{q} \circ \pi_{p, q}\right)(D) \\
& =\left(\bigcirc_{l \in K \backslash\{p\}} \nu_{l} \circ \nu_{q}\right)(D) \\
& \neq \nu(D) \\
& =\left(\nu \circ \pi_{p, q}\right)(D) .
\end{aligned}
$$

This yields $\pi_{p, q} \circ \nu \neq \nu \circ \pi_{p, q}$, and thus, $Z(\Gamma)=\Gamma^{\tau}$.

The construction of the group $\Gamma$ extends that given in Fuchs \& Schmidt [3] for the case $d=2$.

In addition to the subgroups $\Gamma^{\pi}, \Gamma^{\nu}$ and $\Gamma^{\tau}$ considered before, we define the set

$$
\Gamma^{\pi, \tau}:=\left\{\gamma \in \Gamma \mid \gamma=\pi \circ \varphi \text { for some } \pi \in \Gamma^{\pi} \text { and some } \varphi \in \Gamma^{\tau}\right\}
$$


which is of decisive importance in Sections 5 and 6. Since $\Gamma^{\tau}$ is the center of $\Gamma$, we have $\Gamma^{\pi, \tau}=\left\{\gamma \in \Gamma \mid \gamma=\varphi \circ \pi\right.$ for some $\varphi \in \Gamma^{\tau}$ and some $\left.\pi \in \Gamma^{\pi}\right\}$, and this implies that $\Gamma^{\pi, \tau}$ is a subgroup of $\Gamma$.

As a counterpart to the total reflection $\tau$, we denote by

$$
\psi:=\bigodot_{i=1}^{d-1} \pi_{i, i+1}
$$

the total permutation, and consider the sets

$$
\begin{aligned}
\Gamma^{\psi} & :=\left\{\gamma \in \Gamma \mid \gamma=\bigcirc_{l=1}^{m} \psi \text { for some } m \in\{1, \ldots, d\}\right\} \\
\Gamma^{\psi, \nu} & :=\left\{\gamma \in \Gamma \mid \gamma=\varphi \circ \nu \text { for some } \varphi \in \Gamma^{\psi} \text { and some } \nu \in \Gamma^{\nu}\right\} \\
\Gamma^{\psi, \tau} & :=\left\{\gamma \in \Gamma \mid \gamma=\varphi_{1} \circ \varphi_{2} \text { for some } \varphi_{1} \in \Gamma^{\psi} \text { and some } \varphi_{2} \in \Gamma^{\tau}\right\} .
\end{aligned}
$$

Lemma 3.9. $\Gamma^{\psi}, \Gamma^{\psi, \nu}$ and $\Gamma^{\psi, \tau}$ are subgroups of $\Gamma$.

Pro of. It follows from $\bigcirc_{i=1}^{d} \psi=\iota$ that $\Gamma^{\psi}$ is a subgroup of $\Gamma$. Since

$$
\nu_{m}=\bigcirc_{l=1}^{d-(m-1)} \psi \circ \nu_{1} \circ \bigcirc_{l=1}^{m-1} \psi
$$

for all $m \in\{1, \ldots, d\}$, and thus

$$
\nu_{p} \circ \bigcirc_{l=1}^{q-p} \psi=\bigcirc_{l=1}^{q-p} \psi \circ \nu_{q}
$$

for all $p, q \in\{1, \ldots, d\}$ such that $p \leq q$, we also obtain that $\Gamma^{\psi, \nu}$ is a subgroup of $\Gamma$. That $\Gamma^{\psi, \tau}$ is a subgroup of $\Gamma$ follows from the fact that $\Gamma^{\tau}$ is the center of $\Gamma$.

Moreover, $\Gamma^{\pi, \tau} \cap \Gamma^{\psi, \nu}=\Gamma^{\psi, \tau}, \Gamma^{\pi} \cap \Gamma^{\psi, \tau}=\Gamma^{\psi}, \Gamma^{\psi, \tau} \cap \Gamma^{\nu}=\Gamma^{\tau}$ and $\Gamma^{\psi} \cap \Gamma^{\tau}=\{\iota\}$. 
The following lemma, which will be needed in Section 6 below, discusses the transformations in $\Gamma$ with regard to convexity, and its proof is straightforward.

Lemma 3.10. Let $C, D \in \mathcal{C}$ and $a \in(0,1)$. Then the identity

$$
\gamma(a C+(1-a) D)=a \gamma(C)+(1-a) \gamma(D)
$$

holds for every $\gamma \in \Gamma$.

The group $\Gamma$ is a representation of the hyperoctahedral group. This group also has a different representation which has been used by Taylor [5, 6, 7]; see Section 7.

\section{THE TOTAL REFLECTION OF A COPULA}

In this section we define and give an explicit representation of the survival copula of a copula.

For a copula $C \in \mathcal{C}$ the total reflection

$$
\tau(C)
$$

is a copula and is called the survival copula with respect to $C$. The following theorem provides an explicit representation of a reflection of a copula, and it illustrates that the preceding definition of the survival copula is in accordance with that of Durante \& Sempi [1].

Theorem 4.1. Let $\nu \in \Gamma^{\nu}$ such that $\nu=\bigcirc_{l \in K} \nu_{l}$ for some $K \subseteq\{1, \ldots, d\}$ and let $C \in \mathcal{C}$. Then

$$
(\nu(C))(\mathbf{u})=\sum_{L \subseteq K}(-1)^{|K|-|L|} C\left(\boldsymbol{\eta}_{L}\left(\boldsymbol{\eta}_{K}(\mathbf{u}, \mathbf{1}-\mathbf{u}), \mathbf{1}\right)\right) .
$$

In particular,

$$
(\tau(C))(\mathbf{u})=\sum_{L \subseteq\{1, \ldots, d\}}(-1)^{d-|L|} C\left(\boldsymbol{\eta}_{L}(\mathbf{1}-\mathbf{u}, \mathbf{1})\right) .
$$

Pro of. We prove the assertion by induction. Therefore, let $i \in\{1, \ldots, d\}$. Then

$$
\begin{aligned}
\left(\nu_{i}(C)\right)(\mathbf{u}) & =C\left(\boldsymbol{\eta}_{i}(\mathbf{u}, \mathbf{1})\right)-C\left(\boldsymbol{\eta}_{i}(\mathbf{u}, \mathbf{1}-\mathbf{u})\right) \\
& =\sum_{L \subseteq\{i\}}(-1)^{1-|L|} C\left(\boldsymbol{\eta}_{L}\left(\boldsymbol{\eta}_{i}(\mathbf{u}, \mathbf{1}-\mathbf{u}), \mathbf{1}\right)\right) .
\end{aligned}
$$

Now, let $k \in\{2, \ldots, d\}$ and $M \subseteq\{1, \ldots, d\}$ such that $|M|=k$ and assume that the assertion is fulfilled for all $L \subseteq M$ with $1 \leq|L| \leq k-1$. Without loss of generality, we may assume that $M=\{1, \ldots, k\}$. Then we obtain

$$
\left(\left(\underset{l \in\{1, \ldots, k\}}{\bigcirc} \nu_{l}\right)(C)\right)(\mathbf{u})
$$




$$
\begin{aligned}
& =\left(\left(\underset{l \in\{1, \ldots, k-1\}}{\bigcirc} \nu_{l}\right)\left(\nu_{k}(C)\right)\right)(\mathbf{u}) \\
& =\sum_{L \subseteq\{1, \ldots, k-1\}}(-1)^{k-1-|L|}\left(\nu_{k}(C)\right)\left(\boldsymbol{\eta}_{L}\left(\boldsymbol{\eta}_{\{1, \ldots, k-1\}}(\mathbf{u}, \mathbf{1}-\mathbf{u}), \mathbf{1}\right)\right) \\
& =\sum_{L \subseteq\{1, \ldots, k-1\}}(-1)^{k-1-|L|}\left(\nu_{k}(C)\right)\left(\sum_{l=k}^{d} u_{l} \mathbf{e}_{l}+\sum_{l \in\{1, \ldots, k-1\} \backslash L}\left(1-u_{l}\right) \mathbf{e}_{l}+\sum_{l \in L} 1 \mathbf{e}_{l}\right) \\
& =\sum_{L \subseteq\{1, \ldots, k-1\}}(-1)^{k-1-|L|} C\left(\sum_{l=k+1}^{d} u_{l} \mathbf{e}_{l}+\sum_{l \in\{1, \ldots, k-1\} \backslash L}\left(1-u_{l}\right) \mathbf{e}_{l}+\sum_{l \in L \cup\{k\}} 1 \mathbf{e}_{l}\right) \\
& -\sum_{L \subseteq\{1, \ldots, k-1\}}(-1)^{k-1-|L|} C\left(\sum_{l=k+1}^{d} u_{l} \mathbf{e}_{l}+\sum_{l \in\{1, \ldots, k\} \backslash L}\left(1-u_{l}\right) \mathbf{e}_{l}+\sum_{l \in L} 1 \mathbf{e}_{l}\right) \\
& =\sum_{L \subseteq\{1, \ldots, k-1\}}(-1)^{k-|L \cup\{k\}|} C\left(\sum_{l=k+1}^{d} u_{l} \mathbf{e}_{l}+\sum_{l \in\{1, \ldots, k\} \backslash(L \cup\{k\})}\left(1-u_{l}\right) \mathbf{e}_{l}+\sum_{l \in L \cup\{k\}} 1 \mathbf{e}_{l}\right) \\
& +\sum_{L \subseteq\{1, \ldots, k-1\}}(-1)^{k-|L|} C\left(\sum_{l=k+1}^{d} u_{l} \mathbf{e}_{l}+\sum_{l \in\{1, \ldots, k\} \backslash L}\left(1-u_{l}\right) \mathbf{e}_{l}+\sum_{l \in L} 1 \mathbf{e}_{l}\right) \\
& =\sum_{L \subseteq\{1, \ldots, k\}}(-1)^{k-|L|} C\left(\sum_{l=k+1}^{d} u_{l} \mathbf{e}_{l}+\sum_{l \in\{1, \ldots, k\} \backslash L}\left(1-u_{l}\right) \mathbf{e}_{l}+\sum_{l \in L} 1 \mathbf{e}_{l}\right) \\
& =\sum_{L \subseteq\{1, \ldots, k\}}(-1)^{k-|L|} C\left(\boldsymbol{\eta}_{L}\left(\boldsymbol{\eta}_{\{1, \ldots, k\}}(\mathbf{u}, \mathbf{1}-\mathbf{u}), \mathbf{1}\right)\right) .
\end{aligned}
$$

This completes the proof.

We now introduce two main copulas:

Example 4.2. (1) Fréchet-Hoeffding bound: The function $M: \mathrm{I}^{d} \rightarrow \mathbb{R}$ given by

$$
M(\mathbf{u}):=\min \left\{u_{i} \mid i \in\{1, \ldots, d\}\right\}
$$

is a copula (see Nelsen [4, p. 47]) and satisfies $\gamma(M)=M$ for every $\gamma \in \Gamma^{\pi, \tau}$. In particular, $\tau(M)=M$. Moreover, every copula $C \in \mathcal{C}$ satisfies $C \leq M$.

(2) Product copula: The function $\Pi: \mathrm{I}^{d} \rightarrow \mathbb{R}$ given by

$$
\Pi(\mathbf{u}):=\prod_{i=1}^{d} u_{i}
$$

is a copula (see Nelsen [4, p. 47]) and satisfies $\gamma(\Pi)=\Pi$ for every $\gamma \in \Gamma$.

The following corollary is an immediate consequence of Theorem 4.1 .

Corollary 4.3. Every $\nu \in \Gamma^{\nu} \backslash \Gamma^{\tau}$ satisfies $(\nu(M))(\mathbf{1} / \mathbf{2})=0$ and $(\nu(\Pi))(\mathbf{1} / \mathbf{2})=1 / 2^{d}$. 


\section{SUBGROUPS OF $\Gamma$ WHICH PRESERVE CERTAIN PROPERTIES OF COPULAS}

In this section we focus on subgroups of $\Gamma$ which preserve certain properties of copulas.

A copula $C \in \mathcal{C}$ is said to be symmetric if it satisfies

$$
C\left(\boldsymbol{\eta}_{\{i, i+1\}}\left(\mathbf{u}, u_{i+1} \mathbf{e}_{i}+u_{i} \mathbf{e}_{i+1}\right)\right)=C(\mathbf{u})
$$

for all $\mathbf{u} \in \mathrm{I}^{d}$ and all $i \in\{1, \ldots, d-1\}$. Thus, a copula $C \in \mathcal{C}$ is symmetric if and only if $\pi(C)=C$ for all $\pi \in \Gamma^{\pi}$.

A transformation $\gamma \in \Gamma$ is said to be symmetry preserving if, for any symmetric $C \in \mathcal{C}, \gamma(C)$ is symmetric.

Theorem 5.1. A transformation $\gamma \in \Gamma$ is symmetry preserving if and only if $\gamma \in \Gamma^{\pi, \tau}$.

Proof. It is obvious that every element of $\Gamma^{\pi}$ is symmetry preserving. Moreover, let $\widehat{\gamma} \in \Gamma^{\pi, \tau} \backslash \Gamma^{\pi}$ and $C \in \mathcal{C}$ be symmetric. Then there exists a unique $\widehat{\pi} \in \Gamma^{\pi}$ such that $\widehat{\gamma}=\widehat{\pi} \circ \tau$ and hence

$$
\pi(\widehat{\gamma}(C))=(\pi \circ \widehat{\pi} \circ \tau)(C)=(\tau \circ \pi \circ \widehat{\pi})(C)=\tau(C)=(\tau \circ \widehat{\pi})(C)=(\widehat{\pi} \circ \tau)(C)=\widehat{\gamma}(C)
$$

for all $\pi \in \Gamma^{\pi}$. Thus, every element of $\Gamma^{\pi, \tau}$ is symmetry preserving.

Now, let $\widehat{\gamma} \in \Gamma \backslash \Gamma^{\pi, \tau}$. Then Lemma 3.7 provides the existence of unique $\widehat{\pi} \in \Gamma^{\pi}$ and $\widehat{\nu} \in \Gamma^{\nu} \backslash \Gamma^{\tau}$ such that $\widehat{\gamma}=\widehat{\nu} \circ \widehat{\pi}$ and $\widehat{\nu}=\bigcirc_{l \in K} \nu_{l}$ for some $K \subseteq\{1, \ldots, d\}$ with $1 \leq|K| \leq d-1$. First of all, for every symmetric $C \in \mathcal{C}$ we obtain

$$
\widehat{\gamma}(C)=(\widehat{\nu} \circ \widehat{\pi})(C)=\widehat{\nu}(C)=\left(\bigcirc_{l \in K} \nu_{l}\right)(C) .
$$

Moreover, choose $p \in K$ and $q \in\{1, \ldots, d\} \backslash K$. Lemma 3.5 then yields

$$
\begin{aligned}
\left(\pi_{p, q} \circ \widehat{\gamma}\right)(C) & =\left(\pi_{p, q} \circ \bigcirc_{l \in K} \nu_{l}\right)(C) \\
& =\left(\pi_{p, q} \circ \bigcirc_{l \in K \backslash\{p\}} \nu_{l} \circ \nu_{p}\right)(C) \\
& =\left(\bigcirc_{l \in K \backslash\{p\}} \nu_{l} \circ \pi_{p, q} \circ \nu_{p}\right)(C) \\
& =\left(\bigcirc_{l \in K \backslash\{p\}} \nu_{l} \circ \nu_{q} \circ \pi_{p, q}\right)(C) \\
& =\left(\underset{l \in K \backslash\{p\}}{\left.\bigcirc_{l} \circ \nu_{q}\right)(C)}\right.
\end{aligned}
$$

for every symmetric $C \in \mathcal{C}$. Further, consider the copula $D$ discussed in Example 3.3 . We then obtain

$$
\left(\pi_{p, q} \circ \widehat{\gamma}\right)(D)=\left(\bigcirc_{l \in K \backslash\{p\}} \nu_{l} \circ \nu_{q}\right)(D) \neq\left(\bigcirc_{l \in K} \nu_{l}\right)(D)=\widehat{\gamma}(D) .
$$

Thus, the elements of $\Gamma^{\pi, \tau}$ are the only symmetry preserving symmetries.

A transformation $\gamma \in \Gamma$ is said to be order preserving if, for any $C, D \in \mathcal{C}, C \leq D$ implies $\gamma(C) \leq \gamma(D)$. 
Theorem 5.2. (1) Let $d=2$. Then a transformation $\gamma \in \Gamma$ is order preserving if and only if $\gamma \in \Gamma^{\pi, \tau}$.

(2) Let $d \geq 3$. Then a transformation $\gamma \in \Gamma$ is order preserving if and only if $\gamma \in \Gamma^{\pi}$.

Pro of. First of all, assertion (1) follows immediately from Fuchs \& Schmidt [3].

Now, we want to prove (2). It is obvious that every element of $\Gamma^{\pi}$ is order preserving. Moreover, let $\gamma \in \Gamma \backslash \Gamma^{\pi, \tau}$. Then Lemma 3.7 provides the existence of unique $\pi \in \Gamma^{\pi}$ and $\nu \in \Gamma^{\nu} \backslash \Gamma^{\tau}$ such that $\gamma=\nu \circ \pi$. The copulas $M$ and $\Pi$ satisfy $\gamma(\Pi)=\Pi$ and $\gamma(M)=(\nu \circ \pi)(M)=\nu(M)$ as well as $\Pi \leq M$. However, Corollary 4.3 provides

$$
(\gamma(M))(\mathbf{1} / \mathbf{2})=(\nu(M))(\mathbf{1} / \mathbf{2})=0<\frac{1}{2^{d}}=\Pi(\mathbf{1} / \mathbf{2})=(\gamma(\Pi))(\mathbf{1} / \mathbf{2}) .
$$

Thus, the elements of $\Gamma \backslash \Gamma^{\pi, \tau}$ are not order preserving.

Furthermore, let $d$ be odd and $\gamma \in \Gamma^{\pi, \tau} \backslash \Gamma^{\pi}$. Then there exists a unique $\pi \in \Gamma^{\pi}$ such that $\gamma=\tau \circ \pi$. Consider now the function $D: \mathrm{I}^{d} \rightarrow \mathbb{R}$ given by

$$
D(\mathbf{u}):=\prod_{i=1}^{d} u_{i}+\prod_{i=1}^{d} u_{i}\left(1-u_{i}\right)
$$

$D$ is a copula (see Dolati \& Úbeda-Flores [2, Example 4.5]) that is symmetric and satisfies

$$
(\tau(D))(\mathbf{u})=\prod_{i=1}^{d} u_{i}+(-1)^{d} \prod_{i=1}^{d} u_{i}\left(1-u_{i}\right) .
$$

We then have $\Pi \leq D$ but $\gamma(D)=(\tau \circ \pi)(D)=\tau(D) \leq \Pi=\gamma(\Pi)$. Thus, the elements of $\Gamma^{\pi}$ are the only order preserving symmetries.

Finally, let $d$ be even and $\gamma \in \Gamma^{\pi, \tau} \backslash \Gamma^{\pi}$. Then there exists a unique $\pi \in \Gamma^{\pi}$ such that $\gamma=\tau \circ \pi$. For $j \in\{1, \ldots, d\}$, consider the copula

$$
D_{j}(\mathbf{u}):=\prod_{i=1}^{d} u_{i}+\prod_{i=1, i \neq j}^{d}\left(1-u_{i}\right) \prod_{i=1}^{d} u_{i}
$$

discussed in the proof of Theorem 3.1. Then, there exists some $k \in\{1, \ldots, d\}$ such that $\pi\left(D_{j}\right)=D_{k}$ and $D_{k}$ satisfies

$$
\left(\nu_{l}\left(D_{k}\right)\right)(\mathbf{u})=\prod_{i=1}^{d} u_{i}-\prod_{i=1, i \neq k}^{d}\left(1-u_{i}\right) \prod_{i=1}^{d} u_{i}
$$

for all $l \neq k$ and $\nu_{k}\left(D_{k}\right)=D_{k}$. Furthermore,

$$
\left(\tau\left(D_{k}\right)\right)(\mathbf{u})=\prod_{i=1}^{d} u_{i}+(-1)^{d-1} \prod_{i=1, i \neq k}^{d}\left(1-u_{i}\right) \prod_{i=1}^{d} u_{i} .
$$

We then have $\Pi \leq D_{j}$ but $\gamma\left(D_{j}\right)=(\tau \circ \pi)\left(D_{j}\right)=\tau\left(D_{k}\right) \leq \Pi=\gamma(\Pi)$. Thus, the elements of $\Gamma^{\pi}$ are the only order preserving symmetries. 
For $C, D \in \mathcal{C}$ we write $C \preceq D$ if $C \leq D$ and $\tau(C) \leq \tau(D)$. Then $\preceq$ is an order relation and is called concordance order. Obviously, every copula $C \in \mathcal{C}$ satisfies $C \preceq M$.

A transformation $\gamma \in \Gamma$ is said to be concordance order preserving if, for any $C, D \in \mathcal{C}$, $C \preceq D$ implies $\gamma(C) \preceq \gamma(D)$.

Theorem 5.3. A transformation $\gamma \in \Gamma$ is concordance order preserving if and only if $\gamma \in \Gamma^{\pi, \tau}$

Proof. It is obvious that every element of $\Gamma^{\pi, \tau}$ is concordance order preserving. Now, let $\gamma \in \Gamma \backslash \Gamma^{\pi, \tau}$. Then Lemma 3.7 provides the existence of unique $\pi \in \Gamma^{\pi}$ and $\nu \in \Gamma^{\nu} \backslash \Gamma^{\tau}$ such that $\gamma=\nu \circ \pi$. The copulas $M$ and $\Pi$ satisfy $\gamma(\Pi)=\Pi$ and $\gamma(M)=(\nu \circ \pi)(M)=$ $\nu(M)$ as well as $\Pi \preceq M$. However, Corollary 4.3 provides

$$
(\gamma(M))(\mathbf{1} / \mathbf{2})=(\nu(M))(\mathbf{1} / \mathbf{2})=0<\frac{1}{2^{d}}=\Pi(\mathbf{1} / \mathbf{2})=(\gamma(\Pi))(\mathbf{1} / \mathbf{2}) .
$$

Thus, the elements of $\Gamma^{\pi, \tau}$ are the only concordance order preserving symmetries.

Remark 5.4. For the case $d=2$ pointwise order is the same as concordance order. Thus, the subgroup of all order preserving symmetries and the subgroup of all concordance order preserving symmetries coincide as stated in Theorems 5.2 and 5.3

\section{MEASURES OF CONCORDANCE}

In this section, we study the group $\Gamma$ with respect to measures of concordance.

There is a rich literature on measures of concordance for multivariate copulas. For example, Taylor [5, 6, 7] and Dolati \& Úbeda-Flores [2] proposed definitions of a measure of concordance which are closely related. Taylor's definition involves the group $(\tilde{\Gamma}, \diamond)$ of transformations on the unit hypercube (see Section 7), and that of Dolati and ÚbedaFlores employs the partial reflections of a copula.

In the spirit of Taylor [5, 6, 7] and Dolati \& Úbeda-Flores 2, we propose here a quite general definition of a measure of concordance for multivariate copulas, which is based on the group $(\Gamma, \circ)$ :

A function $\kappa: \mathcal{C} \rightarrow[-1,1]$ is said to be a measure of concordance of degree $d$ if it has the following properties:

(i) $\kappa(M)=1$.

(ii) The identity $\kappa(\pi(C))=\kappa(C)$ holds for every $C \in \mathcal{C}$ and every $\pi \in \Gamma^{\pi}$.

(iii) The identity $\kappa(\tau(C))=\kappa(C)$ holds for every $C \in \mathcal{C}$.

(iv) The identity

$$
\sum_{\nu \in \Gamma^{\nu}} \kappa(\nu(C))=0
$$

holds for every $C \in \mathcal{C}$. 
Property (iv) implies that $\kappa(\Pi)=0$.

Except for these basic properties of a measure of concordance, many authors require additional properties. Among those, monotonicity and continuity are the most prominent ones. Some authors like Taylor [5, 6, 7, and Dolati \& Úbeda-Flores 2, also consider consistency of measures of concordance for copulas of different dimensions. For the purpose of the following discussion of measures of concordance, however, we do not need any of these additional properties, and this is why we omit them here although they might be most useful in a different context.

The previous definition of a measure of concordance is in accordance with that given in Fuchs \& Schmidt [3] for $d=2$ : In that case, we have $\nu_{2}=\tau \circ \nu_{1}$, which yields

$$
\begin{aligned}
0 & =\kappa(C)+\kappa\left(\nu_{1}(C)\right)+\kappa\left(\nu_{2}(C)\right)+\kappa(\tau(C)) \\
& =\kappa(C)+\kappa\left(\nu_{1}(C)\right)+\kappa\left(\tau\left(\nu_{1}(C)\right)\right)+\kappa(\tau(C)) \\
& =\kappa(C)+\kappa\left(\nu_{1}(C)\right)+\kappa\left(\nu_{1}(C)\right)+\kappa(C)
\end{aligned}
$$

and hence $\kappa\left(\nu_{1}(C)\right)=-\kappa(C)$ for all $C \in \mathcal{C}$.

The map $\beta: \mathcal{C} \rightarrow[-1,1]$ given by

$$
\beta(C):=\frac{1}{2^{d-1}-1}\left(2^{d-1}[C(\mathbf{1} / \mathbf{2})+(\tau(C))(\mathbf{1} / \mathbf{2})]-1\right)
$$

is called Blomqvist's beta. This is the definition proposed by Úbeda-Flores [8]. The following result is essentially due to Taylor [5].

Lemma 6.1. Blomqvist's beta is a measure of concordance.

Proof. It is obvious that $\beta$ fulfills properties (i),(ii) and (iii). We now prove (iv). To this end, consider $C \in \mathcal{C}$ and put $D:=1 / 2^{d} \sum_{\nu \in \Gamma^{\nu}} \nu(C)$. Since $\mathcal{C}$ is convex we have $D \in \mathcal{C}$. Moreover, with regard to Lemma $3.10 D$ satisfies $\nu_{i}(D)=D$ for all $i \in\{1, \ldots, d\}$ which yields $D(\mathbf{1} / \mathbf{2})=\left(\nu_{i}(D)\right)(\mathbf{1} / \mathbf{2})=D\left(\boldsymbol{\eta}_{i}(\mathbf{1} / \mathbf{2}, \mathbf{1})\right)-D(\mathbf{1} / \mathbf{2})$, and thus, $2 D(\mathbf{1} / \mathbf{2})=D\left(\boldsymbol{\eta}_{i}(\mathbf{1} / \mathbf{2}, \mathbf{1})\right)$ for all $i \in\{1, \ldots, d\}$. Induction then yields $2^{d} D(\mathbf{1} / \mathbf{2})=1$ and hence

$$
\sum_{\nu \in \Gamma^{\nu}}(\nu(C))(\mathbf{1} / \mathbf{2})=1
$$

We further obtain

$$
\begin{aligned}
\sum_{\nu \in \Gamma^{\nu}} \beta(\nu(C)) & =\sum_{\nu \in \Gamma^{\nu}} \frac{1}{2^{d-1}-1}\left(2^{d-1}[(\nu(C))(\mathbf{1} / \mathbf{2})+((\tau \circ \nu)(C))(\mathbf{1} / \mathbf{2})]-1\right) \\
& =\frac{1}{2^{d-1}-1}\left(2^{d-1}\left[\sum_{\nu \in \Gamma^{\nu}}(\nu(C))(\mathbf{1} / \mathbf{2})+\sum_{\nu \in \Gamma^{\nu}}((\tau \circ \nu)(C))(\mathbf{1} / \mathbf{2})\right]-2^{d}\right) \\
& =\frac{1}{2^{d-1}-1}\left(2^{d-1}(1+1)-2^{d}\right)
\end{aligned}
$$




$$
=0 \text {. }
$$

This proves the assertion.

A transformation $\gamma \in \Gamma$ is said to be concordance preserving if it satisfies $\kappa(\gamma(C))=$ $\kappa(C)$ for every measure of concordance $\kappa$ and every $C \in \mathcal{C}$.

Theorem 6.2. A transformation $\gamma \in \Gamma$ is concordance preserving if and only if $\gamma \in \Gamma^{\pi, \tau}$.

Proof. It is obvious that every element of $\Gamma^{\pi, \tau}$ is concordance preserving. Now, consider $\gamma \in \Gamma \backslash \Gamma^{\pi, \tau}$ and note that

$$
\beta(M)=1 .
$$

We want to prove that $\beta(\gamma(M)) \neq 1$. First of all, Lemma 3.7 provides the existence of unique $\pi \in \Gamma^{\pi}$ and $\nu \in \Gamma^{\nu} \backslash \Gamma^{\tau}$ such that $\gamma=\nu \circ \pi$. Moreover, since $\tau$ is in the center of $\Gamma$ we have

$$
(\tau \circ \gamma)(M)=(\gamma \circ \tau)(M)=\gamma(M)=(\nu \circ \pi)(M)=\nu(M)
$$

and Corollary 4.3 provides

$$
((\tau \circ \gamma)(M))(\mathbf{1} / \mathbf{2})=(\gamma(M))(\mathbf{1} / \mathbf{2})=(\nu(M))(\mathbf{1} / \mathbf{2})=0 .
$$

We further obtain

$$
\begin{aligned}
\beta(\gamma(M)) & =\frac{1}{2^{d-1}-1}\left(2^{d-1}[(\gamma(M))(\mathbf{1} / \mathbf{2})+((\tau \circ \gamma)(M))(\mathbf{1} / \mathbf{2})]-1\right) \\
& =-\frac{1}{2^{d-1}-1} .
\end{aligned}
$$

Thus, the elements of $\Gamma^{\pi, \tau}$ are the only concordance preserving symmetries.

Theorem 6.2 emphasizes once more the particular role of the transformations which belong to the subgroup $\Gamma^{\pi, \tau}$ : They are the only concordance preserving symmetries and, as noted in Section 5 the only symmetry and concordance order preserving symmetries.

\section{ANOTHER POINT OF VIEW}

Until now, we have deliberately avoided the use of results or even notions from measure and probability theory. In the present section we sketch, without going too far into the details, a measure theoretic and probabilistic approach which provides some additional insights into the properties of the group of transformations.

First of all, we note that the hyperoctahedral group has another representation which is geometric and quite popular: Consider the collection of all transformations $\mathrm{I}^{d} \rightarrow \mathrm{I}^{d}$ equipped with the composition $\diamond$ and the identity $\tilde{\iota}$. Then, proceeding as in Section 3 , one can show that there is a smallest group $(\tilde{\Gamma}, \diamond)$ containing the transformations $\tilde{\pi}_{i, j}$ : $\mathrm{I}^{d} \rightarrow \mathrm{I}^{d}$ and $\tilde{\nu}_{k}: \mathrm{I}^{d} \rightarrow \mathrm{I}^{d}$ with $i, j, k \in\{1, \ldots, d\}$ and $i \neq j$, given by 


$$
\begin{aligned}
\tilde{\pi}_{i, j}(\mathbf{u}) & :=\boldsymbol{\eta}_{\{i, j\}}\left(\mathbf{u}, u_{j} \mathbf{e}_{i}+u_{i} \mathbf{e}_{j}\right) \\
\tilde{\nu}_{k}(\mathbf{u}) & :=\boldsymbol{\eta}_{k}(\mathbf{u}, \mathbf{1}-\mathbf{u}) .
\end{aligned}
$$

The groups $(\Gamma, \circ)$ and $(\tilde{\Gamma}, \diamond)$ are related to each other by the following result:

Theorem 7.1. There exists a unique isomorphism $T:(\Gamma, \circ) \rightarrow(\tilde{\Gamma}, \diamond)$ satisfying $T\left(\pi_{i, j}\right)$ $=\tilde{\pi}_{i, j}$ and $T\left(\nu_{k}\right)=\tilde{\nu}_{k}$ for all $i, j, k \in\{1, \ldots, d\}$ such that $i \neq j$.

To simplify the notation, we put $\tilde{\gamma}:=T(\gamma)$ for every $\gamma \in \Gamma$.

Since the transformations in $\tilde{\Gamma}$ are continuous and hence measureable, they can be used to transform probability measures on the Borel $\sigma$-field $\mathcal{B}\left(\mathrm{I}^{d}\right)$ or random vectors from some probability space into the measureable space $\left(\mathrm{I}^{d}, \mathcal{B}\left(\mathrm{I}^{d}\right)\right)$.

Let us first consider transformations of probability measures. A probability measure $Q: \mathcal{B}\left(\mathrm{I}^{d}\right) \rightarrow \mathrm{I}$ is said to be a copula measure if the identity $Q\left[\times_{i=1}^{d} B_{i}\right]=\boldsymbol{\lambda}\left[B_{j}\right]$ holds for every collection of Borel sets $\left\{B_{i}\right\}_{i \in\{1, \ldots, d\}} \subseteq \mathcal{B}(\mathrm{I})$ satisfying $B_{i}=\mathrm{I}$ for all $i \in\{1, \ldots, d\} \backslash\{j\}$; here $\boldsymbol{\lambda}$ denotes the Lebesgue measure on $\mathcal{B}(\mathrm{I})$. Let $\mathcal{Q}$ denote the collection of all copula measures on $\mathcal{B}\left(\mathrm{I}^{d}\right)$. The following result, which relies on the measure extension theorem, is a refinement of the correspondence theorem relating distribution functions on $\mathbb{R}^{d}$ to probability measures on $\mathcal{B}\left(\mathbb{R}^{d}\right)$ :

Theorem 7.2. There exists a one-to-one correspondence between $\mathcal{C}$ and $\mathcal{Q}$. Moreover, every corresponding pair of a copula $C \in \mathcal{C}$ and a copula measure $Q \in \mathcal{Q}$ satisfies $C(\mathbf{u})=Q[[\mathbf{0}, \mathbf{u}]]$ for all $\mathbf{u} \in \mathrm{I}^{d}$.

It can also be shown that the collection $\mathcal{Q}$ is stable under transformations of the group $\tilde{\Gamma}$ :

Theorem 7.3. Let $Q \in \mathcal{Q}$ be a copula measure. Then, for every $\tilde{\gamma} \in \tilde{\Gamma}$, the image measure $Q_{\tilde{\gamma}}$ of $Q$ under $\tilde{\gamma}$ satisfies $Q_{\tilde{\gamma}} \in \mathcal{Q}$.

Let us now consider transformations of random vectors. The following result, which relies on Theorems 7.1 and 7.2 , asserts that, for every $\gamma \in \Gamma$, the transformation $\gamma(C)$ of a copula $C \in \mathcal{C}$ is precisely the distribution function of the transformation $\tilde{\gamma} \circ \mathbf{U}$ of a random vector $\mathbf{U}$ with distribution function $C$ :

Theorem 7.4. Let $C \in \mathcal{C}$ be a copula with copula measure $Q \in \mathcal{Q}$. Then $\left(\mathrm{I}^{d}, \mathcal{B}\left(\mathrm{I}^{d}\right), Q\right)$ is a probability space and the identity map $\mathbf{U}:\left(\mathrm{I}^{d}, \mathcal{B}\left(\mathrm{I}^{d}\right), Q\right) \rightarrow\left(\mathrm{I}^{d}, \mathcal{B}\left(\mathrm{I}^{d}\right)\right)$ is a random vector which satisfies $Q[\{\mathbf{U} \leq \mathbf{u}\}]=C(\mathbf{u})$ for all $\mathbf{u} \in \mathrm{I}^{d}$. Moreover, the identity

$$
(\gamma(C))(\mathbf{u})=Q[\{\tilde{\gamma} \circ \mathbf{U} \leq \mathbf{u}\}]
$$

holds for all $\mathbf{u} \in \mathrm{I}^{d}$ and all $\gamma \in \Gamma$.

By Theorem 3.8, we know that $\gamma(C)$ is a copula. This fact could also be established by using first Theorem 7.3 to prove that $Q_{\tilde{\gamma}}$ is a copula measure, then Theorem 7.2 to prove that the map

$$
\mathbf{u} \mapsto Q[\{\tilde{\gamma} \circ \mathbf{U} \leq \mathbf{u}\}]=Q_{\tilde{\gamma} \circ \mathbf{U}}[[\mathbf{0}, \mathbf{u}]]=\left(Q_{\mathbf{U}}\right)_{\tilde{\gamma}}[[\mathbf{0}, \mathbf{u}]]=Q_{\tilde{\gamma}}[[\mathbf{0}, \mathbf{u}]]
$$


is a copula, and finally the identity of Theorem 7.4 to prove that $\gamma(C)$ is a copula.

In conclusion, the investigation of the group $\Gamma$ acting on $\mathcal{C}$ could also be based on the group $\tilde{\Gamma}$ acting on $\mathrm{I}^{d}$, but as shown above, this approach requires several results from measure and probability theory.

\section{ACKNOWLEDGEMENT}

I would like to thank Klaus D. Schmidt for several useful comments and remarks. I am also grateful to the referees for their suggestions which helped to improve the presentation of the paper.

(Received December 20, 2013)

\section{REFERENCES}

[1] F. Durante and C. Sempi: Copula theory: an introduction. In: Copula Theory and Its Applications (P. Jaworski, F. Durante, W. Härdle, T. Rychlik, eds.), Springer, Berlin, Heidelberg 2010, pp. 3-31.

[2] A. Dolati and M. Úbeda-Flores: On measures of multivariate concordance. J. Probab. Stat. Sci. 4 (2006), 147-163.

[3] S. Fuchs and K. D. Schmidt: Bivariate copulas: Transformations, asymmetry and measure of concordance. Kybernetika 50 (2013), 109-125.

[4] R. B. Nelsen: An Introduction to Copulas. Second Edition. Springer, New York 2006.

[5] M. D. Taylor: Multivariate measures of concordance. Ann. Inst. Statist. Math. 59 (2007), 789-806.

[6] M. D. Taylor: Some properties of multivariate measures of concordance. arXiv:0808.3105 (2008).

[7] M.D. Taylor: Multivariate measures of concordance for copulas and their marginals. arXiv:1004.5023 (2010).

[8] M. Úbeda-Flores: Multivariate versions of Blomqvist's beta and Spearman's footrule. Ann. Inst. Statist. Math. 57 (2005) 781-788.

Sebastian Fuchs, Versicherungsmathematik, Technische Universität Dresden, 01062 Dresden. Germany.

e-mail: sebastian.fuchs1@tu-dresden.de 